\title{
Using citizen science in the photo-identification of adult individuals of an amphibian based on two facial skin features
}

\author{
John Gould ${ }^{\text {Corresp., }}{ }^{\text {, John Clulow }}{ }^{1}$, Simon Clulow ${ }^{2}$ \\ ${ }^{1}$ Environmental and Life Sciences, University of Newcastle, Callaghan, NSW, Australia \\ 2 Department of Biological Sciences, Macquarie University, Sydney, NSW, Australia \\ Corresponding Author: John Gould \\ Email address: john.gould@newcastle.edu.au
}

Among amphibians, adults have traditionally been identified in capture-mark-recapture studies using invasive marking techniques with associated ethical, cost and logistical considerations. However, species in this group may be strong candidates for photoidentification based on natural skin features that removes many of these concerns, with this technique opening up opportunities for citizen scientists to be involved in animal monitoring programs. We investigated the feasibility of using citizen science to distinguish between individuals of an Australian anuran (the sandpaper frog, Lechriodus fletcheri) based on a visual analysis of their natural skin features. We collected photographs of marked individuals in the field over three breeding seasons using a smartphone device. This photo-database was used to create an online survey to determine how easily members of the general public could photo-match individuals by a comparison of two facial skin features; black banding that runs horizontally above the tympanum and a background array of tubercles present in this region. Survey participants were provided with 30 closed, multiple choice questions in which they were asked to match separate images of a query frog from small image pools of potential candidate matches. Participants were consistently able to match individuals with a low matching error rate (mean \pm SD of $26 \pm 5$ ) despite the relatively low quality of photographs taken from a smartphone device in the field, with most query frogs being matched by a majority of participants (mean \pm SD of $86.02 \pm$ $9.52 \%)$. These features were found to be unique and stable among adult males and females. Thus, photo-identification is likely to be a valid, non-invasive method for capturemark-recapture for $L$. fletcheri, and likely many anurans that display similar facial skin features. This may become an important alternative to artificial marking techniques, with the challenges of manual photo-matching reduced by spreading workloads among members of the public that can be recruited online. 
1 Using citizen science in the photo-identification of adult individuals of an amphibian based

2 on two facial skin features

3

4 John Gould ${ }^{1,3}$, John Clulow ${ }^{1}$, Simon Clulow ${ }^{1,2}$

5

$6{ }^{1}$ School of Environmental and Life Sciences, University of Newcastle, Callaghan, New South

7 Wales, Australia

$8 \quad 2$ Department of Biological Sciences, Macquarie University, Sydney, New South Wales Australia 9

10 Corresponding Author:

11 John Gould ${ }^{3}$

12 Email address: john.gould@uon.edu.au 


\section{Abstract}

27

28

Among amphibians, adults have traditionally been identified in capture-mark-recapture studies using invasive marking techniques with associated ethical, cost and logistical considerations. However, species in this group may be strong candidates for photo-identification based on natural skin features that removes many of these concerns, with this technique opening up opportunities for citizen scientists to be involved in animal monitoring programs. We investigated the feasibility of using citizen science to distinguish between individuals of an Australian anuran (the sandpaper frog, Lechriodus fletcheri) based on a visual analysis of their natural skin features. We collected photographs of marked individuals in the field over three breeding seasons using a smartphone device. This photo-database was used to create an online survey to determine how easily members of the general public could photo-match individuals by a comparison of two facial skin features; black banding that runs horizontally above the tympanum and a background array of tubercles present in this region. Survey participants were provided with 30 closed, multiple choice questions in which they were asked to match separate images of a query frog from small image pools of potential candidate matches. Participants were consistently able to match individuals with a low matching error rate (mean \pm SD of $26 \pm 5$ ) despite the relatively low quality of photographs taken from a smartphone device in the field, with most query frogs being matched by a majority of participants (mean \pm SD of $86.02 \pm$ $9.52 \%)$. These features were found to be unique and stable among adult males and females. Thus, photo-identification is likely to be a valid, non-invasive method for capture-markrecapture for L. fletcheri, and likely many anurans that display similar facial skin features. This 
47 may become an important alternative to artificial marking techniques, with the challenges of

48 manual photo-matching reduced by spreading workloads among members of the public that can

49 be recruited online.

Keywords: Amphibian; Anuran; Biometrics; Mark-recapture; Phenotypic appearance; Skin

52 tubercles.

\section{Introduction}

55

Animal biometrics is an emerging field that involves the identification of species or individuals based on their external phenotypic characteristics, including natural markings or color patterns (Kühl \& Burghardt, 2013). It has been used as an effective technique for data collection in ecological procedures such as capture-mark-recapture (CMR), as photographic images of an individual's unique markings can be cross-matched within a photo-database for detection of recapture events (Williams, Nichols \& Conroy, 2002; Pebsworth \& LaFleur, 2014). This process has been particularly useful for monitoring species that cannot be easily captured or artificially tagged for identification purposes (Frisch \& Hobbs, 2007; Arandjelović \& Zisserman, 2011; Hughes \& Burghardt, 2015), and has been applied to a diverse number of taxa including mammals (Karanth \& Nichols, 1998), large fish (Arandjelović \& Zisserman, 2011; Hughes \& Burghardt, 2015), crustaceans (Frisch \& Hobbs, 2007), and herpetofauna (Gardiner et al., 2014). With the increased affordability and use of smartphone devices that are equipped with cameras, as well as the advent of camera trapping technology, individuals can now be photographed under field conditions and differentiated with very little cost, logistics or expertise required (Wagner et al., 2008; Haddock, Kim \& Mukai, 2013; Pebsworth \& LaFleur, 2014). Photo-identification is 
thus becoming an increasingly important ecological tool that is also providing greater opportunities for the use of citizen science in animal monitoring programs (Dickinson et al., 2010).

Nevertheless, some drawbacks have limited the application of photo-based CMR including difficulties in manually processing large image datasets 'by eye', particularly those in which markings are only subtly different between individuals, which becomes a time expensive process vulnerable to misidentifications (Katona \& Beard, 1990; Bolger et al., 2012; Crunchant et al., 2017). Such obstacles can be overcome using computer-vision techniques that use pattern recognition algorithms, such as 'hand-crafted' feature descriptors or deep metric learning, which automatically detect, extract and compare feature information from images uploaded to a photodatabase (Van Tienhoven et al., 2007; Takeki et al., 2016; Treilibs et al., 2016; Crunchant et al., 2017). These techniques have been shown to have high accuracy in animal identification and have led to significant labour savings (Morrison et al., 2011). Despite these benefits, most computer assisted systems are only partially automated and still require some degree of manual image processing (Burghardt, 2008). Instead of returning a definitive 'match' or 'no match' decision, a similarity score is calculated between each image pair, with the strongest matching candidates to the query subsequently needing to be visually inspected in order for a 'true' match to be confirmed. It is thus crucial that the feasibility of manual image matching is validated for species prior to the application of photo-based CMR, even for processes that are to become computer-assisted. This challenge of manual image matching, particularly for large databases, may be overcome through the use of citizen science (e.g., Willi et al., 2019), as the speed of matching can be increased by spreading the workload across a large group of people that can effectively be recruited online from any location. 
Among amphibians, traditional techniques for identifying individuals include the

94

95

96

97

98

99

100

101

102

103

104

105

106

107

108

109

110

111

112

113

114

115

placement of an artificial visual marker, the removal of toe pads or the insertion of dyes and microchip transponders (Turner, 1960; Brown, 1997; Simoncelli et al., 2005; Bainbridge et al., 2015). These are invasive processes that may influence animal survival, require expertise and are relatively expensive, thereby limiting their widespread use among citizen scientists (Reisser et al., 2008; Sacchi et al., 2010). In contrast, photo-identification is being increasingly used to differentiate amphibians at both the species and individual level (Bradfield, 2004; Church et al., 2007; Gamble, Ravela \& Mcgarigal, 2008; Bendik et al., 2013; Sannolo et al., 2016; Konovalov, Jahangard \& Schwarzkopf, 2018), and has been shown to have the capacity to outperform traditional marking techniques (Bendik et al., 2013). Nevertheless, the ability for skin features to be used for identification purposes requires investigation to determine if it is a viable alternative that can be performed by volunteers with little prior expertise.

In this study, we examined the feasibility of using citizen science in the visual identification of adult individuals of our model species, the sandpaper frog (Lechriodus fletcheri) based on two facial skin features (banding patterns and tubercles). We asked anonymous participants from the general public, recruited through social media, to visually examine small image datasets and correctly match images of individuals taken at different points in time under natural field conditions from a smartphone device. The main objectives of the study were to determine i) the potential ease of obtaining sufficiently clear images of individuals from smartphones devices and ii) the scalability of photo-matching as a technique that has the potential to be used in citizen scientist projects to assist in mark-recapture modelling of anuran populations. Additional objectives were to assess i) the level of skin feature variability among adult L. fletcheri individuals, and ii) the stability of skin features in adults over time, as well as to 
116 iii) evaluate the efficacy of using our two facial skin features for accurate photo-identification of

117 an anuran species.

118

119 Material and Methods

120 Study Species

121 Lechriodus fletcheri is a medium sized frog $(4-5 \mathrm{~cm})$ found in montane temperate forests along

122 the east coast of Australia (Clulow \& Swan, 2018). This species has a prolonged breeding season

123 in the austral spring-summer (September-March) with adults congregating at ephemeral pools

124 during periods of heavy rain to reproduce. Both sexes possess unique facial skin features that

125 have the potential to be used for photo-identification (Fig. 1). A region of black banding that runs

126 horizontally above the tympanum from the corner of the eye to the front leg is a strong candidate

127 marker due to its relatively large size and high level of outline variability between individuals.

128 The skin in this region also has many small epithelial projections (tubercles) that form a unique

129 background pattern which might be an effective secondary feature for photo-analysis. An area of

130 facial skin that included both features was selected as our region of interest (ROI) for visual

131 analysis. All other skin features, particularly dorsal, ventral and leg patterns were not found to be

132 distinct and sufficiently clear for photo-identification purposes.

133

134 Frog Capture and Imaging

135 This study was conducted within a localized area of the Watagan Mountains (330 00’30.6 S,

$136151^{\circ} 23^{\prime} 15.7$ E, datum: GDA2020), NSW, Australia. During the 2015/16, 2016/17 and 2017/18

137 breeding seasons, pools located within the study site were routinely surveyed during periods of

138 rainfall for the presence of adult L. fletcheri individuals. Due to the small size of adults, 
139 nocturnal activity and preference for wet conditions, individuals had to be hand captured in order

140 to obtain a sufficiently clear and unobstructed view of the facial skin features.

141 The right ROI of each individual was photographed using an iPhone 6 (Apple

142 Corporation, Cupertino, California, United States) set to manual image capture without flash. To

143 ensure consistency between images, the focal plane of the lens and the lateral side of each frog

144 was kept approximately parallel. This was achieved by gently grasping the back legs so that the

145 thighs were clasped together and the frog kept splayed in a relaxed position, with the index

146 finger kept beneath the belly to keep the body horizontal. Each frog was held approximately 10-

$14720 \mathrm{~cm}$ from the lens with the light of a head lamp shone from above the body of the frog to

148 illuminate the skin while avoiding overexposure. Each frog was marked with a passive integrated

149 transponder (PIT) tag prior to release as a secondary method of confirming animal identification.

150 Tags were placed behind the front leg via subcutaneous injection. Photographs were labelled

151 with each individual's corresponding tag number. Animals were released within a few minutes of

152 point of capture; each photograph was stored in a library along with information pertaining to the

153 date of animal capture. Animals recaptured across each season were processed in the same

154 manner, resulting in a photo-database containing multiple images of individuals recaptured on

155 different dates. In addition, the facial skin features of an adult male and female were

156 photographed using a stereo-microscope mounted DAGE-MTI camera with Leica LAS EZ

157 software V4.0.0 (Leica Microsystems, Wetzlar, Germany), to obtain high resolution images of

158 skin features present within the ROI.

159 
160

161

16

163

164

165

166

167

168

169

170

171

172

173

174

175

176

177

178

179

180

181

182

\section{Analysis of Variability in Skin Features}

The level of feature variability between individuals was assessed by visually comparing banding regions and tubercles present within the ROI between 10 males and 10 females randomly selected from the database. The temporal stability of these features were also assessed by visually comparing the ROI from individuals which were photographed at different points in time, utilizing the 10 individuals with the longest interval between capture events. In all analyses, ROI's were extracted from each image by cropping out unwanted sections of skin and background.

\section{Online Survey Testing Capacity of Participants to Photo-identify Lechriodus fletcheri}

An online survey was developed using the ROI photo-library to determine how easily L. fletcheri adults could be photo-matched from images taken at different times during the breeding season by members of the public. We used Facebook's survey application (Code Rubik Inc, Montreal, Canada) to construct and disseminate the survey. This platform is freely available via any device with access to Facebook and was used given the capacity to reach a large audience with very little cost or logistics. Participation was anonymous and no screening of individuals was performed based on previous photo-matching experience, thereby allowing us to obtain results that would be reflective of the image-matching ability of the general public in a citizen science project.

Our survey consisted of 30 closed, multiple choice questions in which participants were required to match two separate images of the same frog from a pool of images of different frogs. For each question, participants were shown a query image of the right ROI of an individual frog. Six additional ROI images were shown below the query image, five of which were of different 
183 individuals that were not a match, and one that was a match showing the same frog but

184 photographed on a separate occasion. Participants were asked to select the matching image

185 amongst the set of six by comparing the banding pattern and positioning of tubercles within the

186 ROI. A sample image of an L. fletcheri adult was provided prior to the survey with instructions

187 on how to compare skin features between images.

188 The 30 query images were randomly selected from the photo-database, on condition they 189 were of frogs that had been photographed on at least two separate occasions in the field so that matching images of each query frog could be placed into the corresponding answer pool. These

191

192

193

194

196

197

198

199

200

201

202

203

204

205

matching images were randomly selected from the available images of each query frog

remaining within the photo-database. The remaining five non-matching images were also

randomly selected from the photo-database, with each answer pool composed of a different combination of non-matching images. Each question was displayed to participants separately, with the order of questions kept constant for each trial. All six answer images were presented together to reduce primacy effects, in which options presented earlier are more likely to be selected (Krosnick \& Alwin, 1987). The ordering of images within each question changed for each trial. Participants were only allowed to complete the survey once, with incomplete surveys removed from analysis, which was set up as part of the survey design.

We determined the capacity of participants to successfully match different images of the same frog from small image pools based on the number of questions correctly answered, as well as the time required for participants to complete the survey. Variability in rates of correct image matching between query frogs (i.e. rate of successful matching per question) was also examined, to determine whether some frogs were more easily identified than others. We also examined whether the time taken to complete the survey influenced the proportion of query images that 
206 were correctly matched per survey event using a generalized linear mixed effect model

207 (GLMM), with a random intercept to account for differences between participants. Statistical

208 analysis was performed using RStudio version 1.3.959 (RStudio Team, 2020).

Ethics approval

211 This work was conducted under NPWS Scientific license no. SL101991 and approved by the

212 University of Newcastle Human Research Ethics Committee (approval no. H-2019-0091) and the 213 University of Newcastle Animal Care and Ethics Committee (no. A-2011-138). All experimental

214 procedures were performed in accordance with the Australian code for the care and use of

215 animals for scientific purposes

216

\section{Results}

218 A total of 790 photographs were taken over the course of the three breeding seasons. Our database included 606 unique individuals, 15\% of which were recaptured and photographed on more than one occasion. The number of days separating capture events varied from a few days to more than a year. Facial bandings used for photo identification were found to be highly polymorphic between individuals of both sexes, along with background arrays of tubercles (Fig.

2). Both features were also found among all individuals captured and clearly identifiable irrespective of sex or skin colouration. Both skin features were also found to be stable over time, 225 with no apparent change after more than a year (Fig. 3).

227 the field that were then used in the survey was mean $\pm \mathrm{SD}=15.57 \pm 17.90 \mathrm{~d}$. A total of 87

228 anonymous participants completed the survey; the majority were from Australia (75\%) and 
229 accessed the survey from a mobile device (77\%). The mean time required to complete the survey

230 was mean $\pm \mathrm{SD}=14.37 \pm 25.03 \mathrm{~min}$, with $90 \%$ of participants completing the survey within 20

$231 \mathrm{~min}$ (less than one min per question). One participant had an irregularly long survey time $(51 \mathrm{~h})$

232 which was not included in these time estimates.

233 The average number of query images that were correctly matched ranged from $6 / 30$ to

$23430 / 30$, with a mean \pm SD of $26 \pm 5$ (Fig. 4). The number of query images correctly matched by a

235 participant was not related to the amount of time taken to complete the survey (GLMM, $Z_{83}=$ -

$2360.003, P=0.996)$. Some query frogs were matched correctly more often than others (Fig. 5). The

237 lowest rate of successful matching for a frog was $65 \%$ of participants while the highest was

$238100 \%$, with a mean \pm SD of $86.02 \pm 9.52 \%$.

\section{Discussion}

241 We established that using photographs taken in the field to identify and differentiate between

242 adult individuals of an anuran amphibian based solely on their natural skin marking features is

243 feasible, including the ability of this process of photo-matching to be performed by citizen

244 scientist with very little expertise or prior training. We also met essential criteria for the future

245 application of this technique in capture-mark-recapture studies for our model species; namely

246 demonstration of sufficient inter-individual variation in skin marking features so that individuals

247 can be identified with a high degree of accuracy, and temporal stability of those features so that

248 individuals can be re-identified across subsequent recapture events (Pennycuick, 1978; Marshall

$249 \&$ Pierce, 2012).

250

We found a high level of stable inter-individual variation in facial skin features of both

251 male and female $L$. fletcheri adults. While participants were asked to match frogs based on a 
252 combination of both feature types, the most discernible of these was the region of black banding

253 that runs horizontally above the tympanum. Given its irregular shape, this feature is likely to be

254 the most suitable for manual photo-identification for this species and potentially many other

255 anuran amphibians given the widespread occurrence of this skin feature in this group (e.g.,

256 Australian species; Anstis, 2013; Clulow \& Swan, 2018). This facial feature may also be a better

257 candidate for inter-individual character discrimination than others previously examined in

258 anurans amphibians such as dorsal patterns, which may be too complex for visual comparison or

259 completely missing in a proportion of individuals of some species (Kenyon et al., 2009).

260 Differences in the number and positioning of tubercles was also apparent between individuals,

261 though likely more difficult to discern than banding patterns. We suggest that the large number

262 of uniform and repeated features that comprise each array of tubercles across the skin surface

263 would result in a robust visual fingerprint for each individual that would be amendable for

264 computer assisted techniques (Lowe, 1999).

Both facial features in L. fletcheri were temporally stable over at least a 12 month period.

This suggests that both are likely to be genetically determined, albeit influenced by

267 environmental factors during development, and permanent (Murray, 1981; Arntzen \& Wallis,

1999; Hoffman \& Blouin, 2000; Wollenberg et al., 2008), which is critical for studies lasting

multiple breeding seasons. Given the short lifespan of L. fletcheri adults (Gould et al., 2020), it is

difficult to determine whether marking features are stable for longer periods than the recapture 
275 to external sources of variation, such as the method of photographing used. For example, as the

276 skin in the ROI of this species is neither rigid nor flat, the manner in which individuals are held

277 during image acquirement may lead to distortion of features, preventing observers from detecting

278 a true match and leading to possible false rejections. However, this problem was readily

279 mitigated by following a consistent imaging protocol between sampling periods in our study.

280 The capacity for participants from the public to consistently and correctly identify $L$.

281 fletcheri individuals based on our target skin features was supported by the results of the online

282 survey. Anonymous participants from the general public were able to correctly match capture

283 (query) and recapture (answer) images of individuals when provided with small image pools,

284 with a majority of participants correctly matching over $85 \%$ of queries and a majority of survey

285 questions answered correctly more than $80 \%$ of the time. Such rates of successful image

286 classification appear to be comparative to those achieved in the identification of individuals of

287 other animal types (Schofield et al., 2008), and species from large camera surveys (Swanson et

288 al., 2016). Given that participants were provided only minimal training prior to the survey, as

289 well as the short time period taken to answer each question (less than one minute on average),

290 these results suggest that $L$ fletcheri adults can be easily and rapidly photo-identified, at least

291 within small image pools, and that such citizen science programs may be useful in monitoring

292 other amphibian populations.

293

There was, however, variability in matching score rates between query frogs, with some

294 incurring more false matches than others. Although the reasons for this disparity were not

analyzed as part of this study, it is likely that some matches are not as obvious as others, 
298 all individuals, there was also a level of variability in ability for true matches to be detected "by

299 eye'. Misidentifications, even if they occur at a low rate, may result in inaccurate parameter

300 estimates in mark-capture-recapture models (Stevick et al., 2001; Yoshizaki et al., 2009). The

301 probability of incorrect matches could be reduced by providing additional training, particularly

302 in the matching of the background array of tubercles that were more difficult to visually assess,

303 by ensuring all images acquired are of sufficient quality before they are uploaded to the photo-

304 database, or by having multiple observers score images and using consensus scores. Indeed, the

305 impact of variable matching ability among participants was not found to be an issue as all query

306 images were overwhelmingly matched correctly when taking into consideration the populous

307 vote.

308 It is probable that the frequency of correctly photo-matching individuals would decrease

309 as the pool of potential matches increases. Manual photo-matching becomes time consuming and

310 may even become impractical with increasing database size (Katona and Beard, 1990; Speed,

311 Meekan \& Bradshaw, 2007; Bolger et al., 2012), a fact that has restricted the use of this

312 technique to small populations (Karanth \& Nichols, 1998; Langtimm et al., 2004). Under this

313 scenario, automated approaches may be required. However, it is still important to validate the

314 capacity for individuals from a population to be identified manually. This is because partially

315 automated systems may, on some occasions, only be able to reduce the number of potential

316 matching individuals down to a small pool of candidates with similar marking features, which an

317 observer will then have to sort through to select a true match. This is likely to occur in most

318 populations as some individuals would be expected to share some similar features (Ottensmeyer

$319 \&$ Whitehead, 2003), even for those species with high inter-individual marking variability.

320 Validating the ability for correct matches to be manually selected from small image pools is thus 
321 an important first step prior to the establishment of computer-assisted methods; a process we

322 have shown could be performed by citizen scientists in future monitoring programs.

We showed that facial marking features in L. fletcheri adults are individually unique and temporally stable, making them effective for photo-identification for conducting non-invasive capture-mark-recapture studies. This process reduces handling times and circumvents the need for invasive tagging techniques with associated logistical, cost and ethical challenges (Reisser et al., 2008). It also has the added benefit of allowing members of the public to become involved in citizen science and conservation programs, either as individuals who are able to acquire images with just the use of a mobile device (under the strict guidance of scientists present), and those who can analyse the data online on their own at any given time. As an increasing number of species require monitoring for their effective management and funding and resources are becoming increasingly spread thin across conservation programs (Margules and Pressey, 2000; Butchart et al., 2010), reliable but less expensive methods of population monitoring are needed. validation for new species, our results suggest that it is likely to be useful in many other anurans that display distinct facial skin features.

\section{Acknowledgments}

339 We thank L. Bainbridge for her advice throughout this study.

\section{References}

341 Anstis M. 2013. Tadpoles and frogs of Australia. Australia: New Holland Publishers. 
342 Arandjelović R, Zisserman A. 2011. Smooth object retrieval using a bag of boundaries. In 2011

343 IEEE international conference on computer vision, Spain, 375-382 DOI : 10.1109/ICCV.2011.6126265.

345

346

347

348

349

350

351

352

353

354

355

356

357

358

359

360

361

362
Arntzen JW, Wallis GP. 1999. Geographic variation and taxonomy of crested newts (Triturus cristatus superspecies): morphological and mitochondrial DNA data. Contributions to Zoology 68 : 181-203 DOI : 10.1163/18759866-06803004.

Araujo G, Abdul RI, Cat M, David M, Christine GL, Sally S, Jessica L, Mabel M-M, Alessandro P. 2020. Getting the most out of citizen science for endangered species such as Whale Shark. Journal of Fish Biology 96 : 864-867 DOI : 10.1111/jfb.14254.

Bainbridge L, Stockwell M, Valdez J, Klop-Toker K, Clulow S, Clulow J, Mahony M. 2015. Tagging tadpoles: retention rates and impacts of visible implant elastomer (VIE) tags from the larval to adult amphibian stages. The Herpetological Journal 25: 133-140.

Bendik NF, Morrison TA, Gluesenkamp AG, Sanders MS, O’donnell LJ. 2013. Computerassisted photo identification outperforms visible implant elastomers in an endangered salamander, Eurycea tonkawae. PloS one 8 : e59424 DOI : 10.1371/journal.pone.0059424.

Bolger DT, Morrison TA, Vance B, Lee D, Farid H. 2012. A computer-assisted system for photographic mark-recapture analysis. Methods in Ecology and Evolution 3 : 813-822 DOI : 10.1111/j.2041-210X.2012.00212.x.

Bradfield KS. 2004. Photographic identification of individual Archey's frogs, Leiopelma archeyi, from natural markings. New Zealand: Department of Conservation Wellington. 
363 Brown LJ. 1997. An evaluation of some marking and trapping techniques currently used in the study of anuran population dynamics. Journal of Herpetology 31 : 410-419 DOI : $10.2307 / 1565670$.

Burghardt T. 2008. Visual animal biometrics: automatic detection and individual identification by coat pattern. D. Phil. Thesis, University of Bristol.

Butchart SH, Walpole M, Collen B, Van Strien A, Scharlemann JP, Almond RE, ... Watson R. 2010. Global biodiversity: indicators of recent declines. Science 328 : 1164-1168 DOI : 10.1126/science.1187512.

Church DR, Bailey LL, Wilbur HM, Kendall WL, Hines JE. 2007. Iteroparity in the variable environment of the salamander Ambystoma tigrinum. Ecology 88 : 891-903.

Clulow S, Swan M. 2018. A complete guide to frogs of Australia. Australia: Australian Geographic.

Crunchant AS, Egerer M, Loos A, Burghardt T, Zuberbühler K, Corogenes K, Leinert V, Kulik L, Kühl H. 2017. Automated face detection for occurrence and occupancy estimation in chimpanzees. American Journal of Primatology 79 : 1-12 DOI : 10.1002/ajp.22627.

Dickinson JL, Benjamin Z, David NB. 2010. Citizen science as an ecological research tool: challenges and benefits. Annual Review of Ecology, Evolution, and Systematics 41 : 149-172 DOI : 10.1146/annurev-ecolsys-102209-144636.

Frisch AJ, Hobbs J-PA. 2007. Photographic identification based on unique, polymorphic colour patterns: a novel method for tracking a marine crustacean. Journal of Experimental Marine Biology and Ecology 351 : 294-299 DOI : 10.1016/j.jembe.2007.07.008

Gamble L, Ravela S, Mcgarigal K. 2008. Multi-scale features for identifying individuals in large biological databases: an application of pattern recognition technology to the marbled 
salamander Ambystoma opacum. Journal of Applied Ecology 45 : 170-180 DOI :

387 10.1111/j.1365-2664.2007.01368.x.

388

389

390

391

392

393

394

395

396

397

398

399

400

401

402

403

404

405

406

Gardiner RZ, Doran E, Strickland K, Carpenter-Bundhoo L, Frère C. 2014. A face in the crowd: a non-invasive and cost effective photo-identification methodology to understand the fine scale movement of eastern water dragons. PloS one 9 : e96992 DOI : 10.1371/journal.pone.0096992.

Gould J, Clulow J, Clulow S. 2020. Risky business in ephemeral waters: the reproductive ecology of the sandpaper frog, Lechriodus fletcheri. PhD thesis, University of Newcastle, Newcastle.

Haddock LJ, Kim DY, Mukai S. 2013. Simple, inexpensive technique for high-quality smartphone fundus photography in human and animal eyes. Journal of ophthalmology 2013: 1-5 DOI : 10.1155/2013/518479

Hoffman EA, Blouin MS. 2000. A review of colour and pattern polymorphisms in anurans. Biological Journal of the Linnean Society 70: 633-665 DOI : 10.1111/j.10958312.2000.tb00221.x.

Hughes B, Burghardt T. 2015. Affinity matting for pixel-accurate fin shape recovery from great white shark imagery. In T. Amaral, S. Matthews, T. Plötz, S. McKenna, and R. Fisher (Eds.), Proceedings of the Machine Vision of Animals and their Behaviour (MVAB), BMVA Press, UK, 8.1-8.8 DOI : 10.5244/C.29.MVAB.8.

Karanth KU, Nichols JD. 1998. Estimation of tiger densities in India using photographic captures and recaptures. Ecology 79 : 2852-2862 DOI : 10.1017/S1367943004001477. 
407 Katona SK, Beard JA. 1990. Population size, migrations and feeding aggregations of the

408

409

410

411

412

413

414

415

416

417

418

419

420

421

422

423

424

425

426

427

428

429

humpback whale (Megaptera novaeangliae) in the western North Atlantic Ocean. Report of the International Whaling Commission (Special Issue 12) : 295-306.

Kenyon N, Phillott AD, Alford RA. 2009. Evaluation of the photographic identification method (PIM) as a tool to identify adult Litoria genimaculata (Anura: Hylidae). Herpetological Conservation and Biology 4: 403-410.

Konovalov DA, Jahangard S, Schwarzkopf L. In situ cane toad recognition. In 2018. digital image computing: techniques and applications (DICTA), Australia, 1-7 DOI : 10.1109/DICTA.2018.8615780.

Krosnick JA, Alwin DF. 1987. An evaluation of a cognitive theory of response-order effects in survey measurement. Public Opinion Quarterly 51 : 201-219 DOI : 10.1086/269029.

Kühl HS, Burghardt T. 2013. Animal biometrics: quantifying and detecting phenotypic appearance. Trends in Ecology and Evolution $28: 432-441$ DOI : 10.1016/j.tree.2013.02.013.

Langtimm CA, Beck CA, Edwards HH, Fick-Child KJ, Ackerman BB, Barton SL, Barton SL, Hartley WC. 2004. Survival estimates for Florida manatees from the photo-identification of individuals. Marine Mammal Science 20 : 438-463 DOI : 10.1111/j.17487692.2004.tb01171.x.

Lowe DG. 1999. Object recognition from local scale-invariant features. In proceedings of the seventh IEEE international conference on computer vision, Greece, 2: 1150-1157 DOI : 10.1109/ICCV.1999.790410.

Margules CR, Pressey RL. 2000. Systematic conservation planning. Nature 405 : 243-253 DOI : $10.1038 / 35012251$. 
430 Marshall AD, Pierce SJ. 2012. The use and abuse of photographic identification in sharks and

431

432

433

434

435

436

437

438

439

440

441

442

443

444

445

446

447

448

449

450

451 rays. Journal of Fish Biology 80 : 1361-1379 DOI : 10.1111/j.1095-8649.2012.03244.x.

Morrison TA, Yoshizaki J, Nichols JD, Bolger DT. 2011. Estimating survival in photographic capture-recapture studies: overcoming misidentification error. Methods in Ecology and Evolution 2 : 454-463 DOI : 10.1111/j.2041-210X.2011.00106.x.

Murray JD. 1981. On pattern formation mechanisms for lepidopteran wing patterns and mammalian coat markings. Philosophical Transactions of the Royal Society of London. B, Biological Sciences 295 : 473-496 DOI : 10.1098/rstb.1981.0155.

Ottensmeyer CA, Whitehead H. 2003. Behavioural evidence for social units in long-finned pilot whales. Canadian Journal of Zoology 81 : 1327-1338 DOI : 10.1139/z03-127.

Pebsworth PA, Lafleur M. 2014. Advancing primate research and conservation through the use of camera traps: introduction to the special issue. International Journal of Primatology 35 : 825-840 DOI : 10.1007/s10764-014-9802-4.

Pennycuick CJ. 1978. Identification using natural markings. Pp. 147-159 in B. Stonehouse (Ed.), Animal marking: recognition marking of animals in research. Proceedings of the RSPCA Symposium 1977. UK: MacMillan Press.

Reisser J, Proietti M, Kinas P, Sazima I. 2008. Photographic identification of sea turtles: method description and validation, with an estimation of tag loss. Endangered Species Research 5 : 73-82 DOI : 10.3354/esr00113.

Sacchi R, Scali S, Pellitteri-Rosa D, Pupin F, Gentilli A, Tettamanti S, Cavigioli L, Racina L, Maiocchi V, Galeotti P, Fasola M. 2010. Photographic identification in reptiles: a matter of scales. Amphibia-Reptilia 31 : 489-502 DOI : 10.1163/017353710X521546 
452 Sannolo M, Gatti F, Mangiacotti M, Scali S, Sacchi R. 2016. Photo-identification in amphibian

453 studies: a test of I3S Pattern. Acta Herpetologica $11: 63-68$ DOI :

454 10.13128/Acta_Herpetol-17198.

455 Schofield G, Katselidis KA, Dimopoulos P, Pantis JD. 2008. Investigating the viability of photo-

456

457

458

459

460

461

462

463

464

465

466

467

468

469

470

471

472

473

474 identification as an objective tool to study endangered sea turtle populations. Journal of Experimental Marine Biology and Ecology 360: 103-108 DOI: 10.1016/j.jembe.2008.04.005

Simoncelli F, Fagotti A, Dall'olio R, Vagnetti D, Pascolini R, Di Rosa I. 2005. Evidence of Batrachochytrium dendrobatidis infection in water frogs of the Rana esculenta complex in central Italy. EcoHealth 2 : 307-312 DOI : 10.1007/s10393-005-8337-8.

Speed CW, Meekan MG, Bradshaw CJ. 2007. Spot the match-wildlife photo-identification using information theory. Frontiers in Zoology 4 : 2 DOI : 10.1186/1742-9994-4-2.

Stevick PT, Palsbøll PJ, Smith TD, Bravington MV, Hammond PS. 2001. Errors in identification using natural markings: rates, sources, and effects on capture recapture estimates of abundance. Canadian Journal of Fisheries and Aquatic Sciences 58 : 1861-1870 DOI : $10.1139 / \mathrm{f01}-131$

Swanson A, Kosmala M, Lintott C, Packer C. 2016. A generalized approach for producing, quantifying, and validating citizen science data from wildlife images. Conservation Biology 30: 520-531 DOI: 10.1111/cobi.12695

Takeki A, Trinh TT, Yoshihashi R, Kawakami R, Iida M, Naemura T. 2016. Detection of small birds in large images by combining a deep detector with semantic segmentation. In 2016 IEEE international conference on image processing (ICIP), USA, 3977-3981 DOI : 10.1109/ICIP.2016.7533106. 
475 RStudio Team. 2020. RStudio: integrated development for R. Boston: RStudio, Inc.

476 Treilibs CE, Pavey CR, Hutchinson MN, Bull CM. 2016. Photographic identification of

477 individuals of a free-ranging, small terrestrial vertebrate. Ecology and Evolution $6: 800$ -

$478 \quad 809$ DOI : $10.1002 /$ ece 3.1883

479 Turner FB. 1960. Population structure and dynamics of the western spotted frog, Rana $p$.

480 pretiosa Baird \& Girard, in Yellowstone Park, Wyoming. Ecological Monographs 30 :

481 251-278 DOI : $10.2307 / 1943562$

482

483

484

485

486

487

488

489

490

491

492

493

494

495

496

497

Van Tienhoven, AM, Den Hartog JE, Reijns RA, Peddemors VM. 2007. A computer-aided program for pattern-matching of natural marks on the spotted raggedtooth shark Carcharias taurus. Journal of Applied Ecology 44 : 273-280 DOI : 10.1111/j.13652664.2006.01273.x.

Wagner D, Reitmayr G, Mulloni A, Drummond T, Schmalstieg D. 2008. Pose tracking from natural features on mobile phones. In Proceedings of the 7th IEEE/ACM international symposium on mixed and augmented reality, UK, 125-134 DOI : 10.1109/ISMAR.2008.4637298.

Williams BK, Nichols JD, Conroy MJ. 2002. Analysis and management of animal populations. USA: Academic Press.

Willi M, Pitman RT, Cardoso AW, Locke C, Swanson A, Boyer A, Veldthuis M, Fortson L. 2019. Identifying animal species in camera trap images using deep learning and citizen science.

Methods in Ecology and Evolution 10: 80-91 DOI: 10.1111/2041-210X.13099

Wollenberg KC, Lötters S, Mora-Ferrer C, Veith M. 2008. Disentangling composite colour patterns in a poison frog species. Biological Journal of the Linnean Society 93 : 433-444 DOI : 10.1111/j.1095-8312.2007.00906.x 
498 Yoshizaki J, Pollock KH, Brownie C, Webster RA. 2009. Modeling misidentification errors in 499 capture-recapture studies using photographic identification of evolving marks. Ecology $500 \quad 90: 3-9$ DOI $: 10.1890 / 08-0304.1$

501 
Figure 1

Target facial skin features of adult Lechriodus fletcheri.

Microscope images of natural skin features present within the chosen region of interest of an male (A) and female (B). Scale bar $=5 \mathrm{~mm}$.
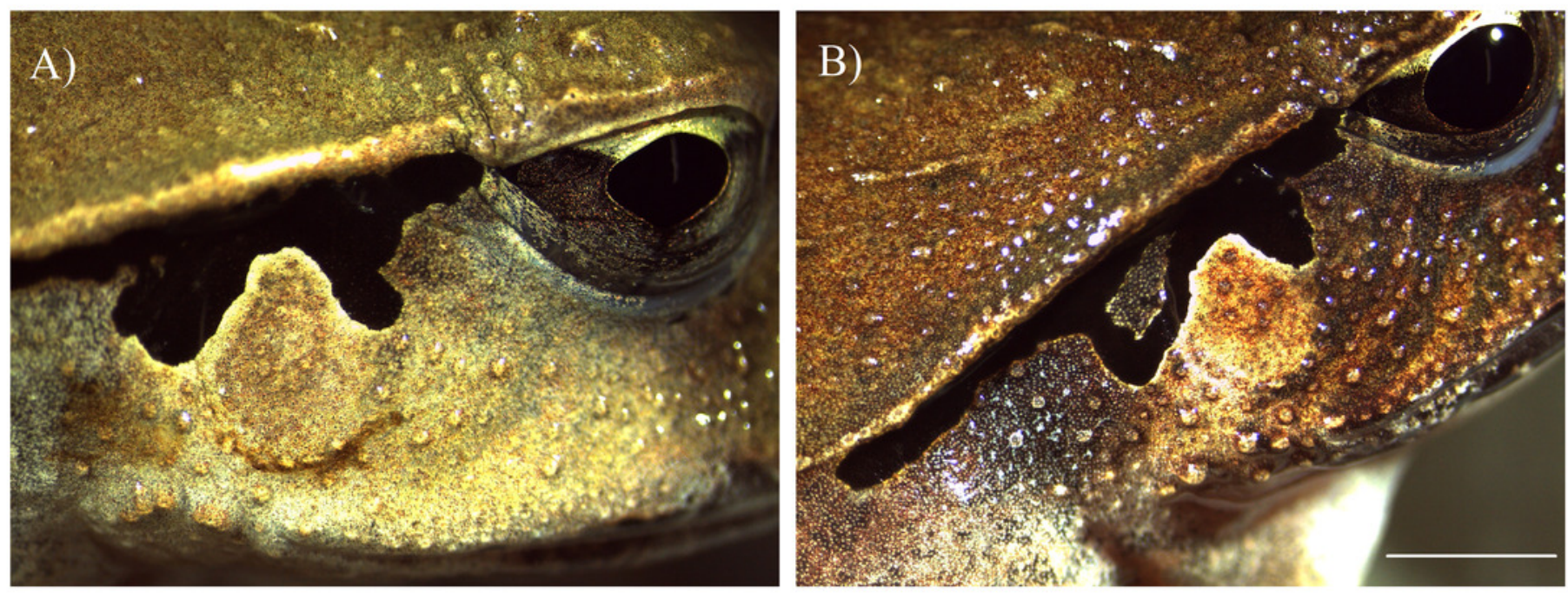
Figure 2

Intra-specific variability in banding patterns among adult Lechriodus fletcheri individuals.

Banding patterns of 10 adults of both sexes are shown (A-J $=$ males; $1-10=$ females).

Banding derived from black and white photographs with background information removed.

A)

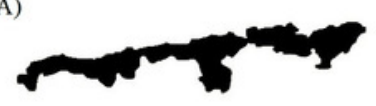

C)

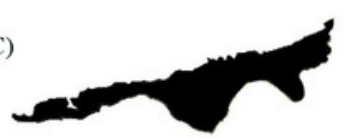

E)

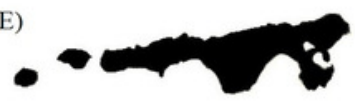

G)
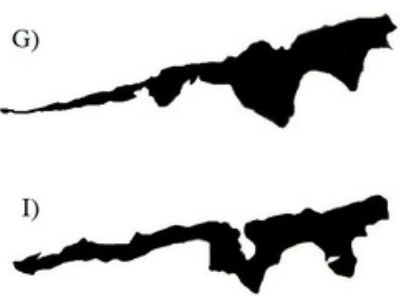

B)

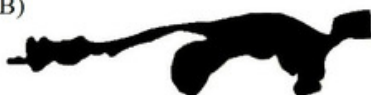

D)

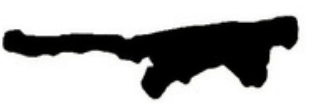

F)

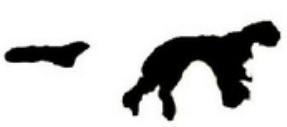

$\mathrm{H})$
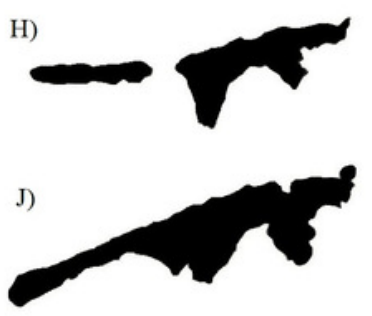

1)

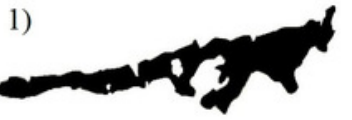

3) rat

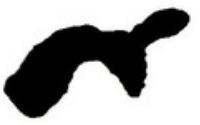

5)

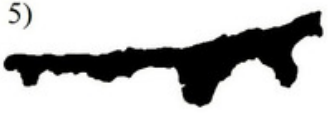

7)

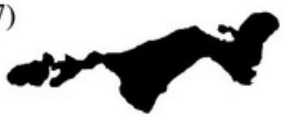

9)

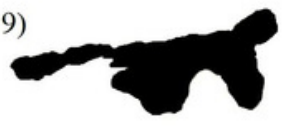

2)

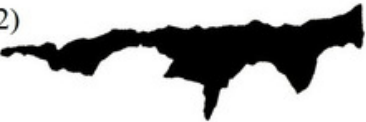

4)

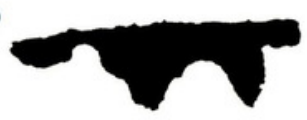

6)

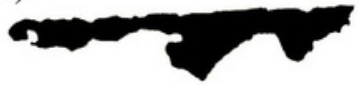

8)

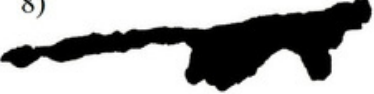

10)

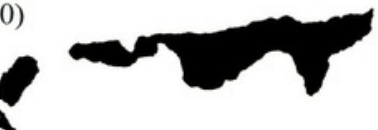




\section{Figure 3}

Photographs of four adult Lechriodus fletcheri individuals over time highlighting the stability of natural skin features.

Images on the left taken at time of first capture, images on the right taken at time of recapture (A) 299, B) 383, C) 86, and D) 123 days post-capture).

A)
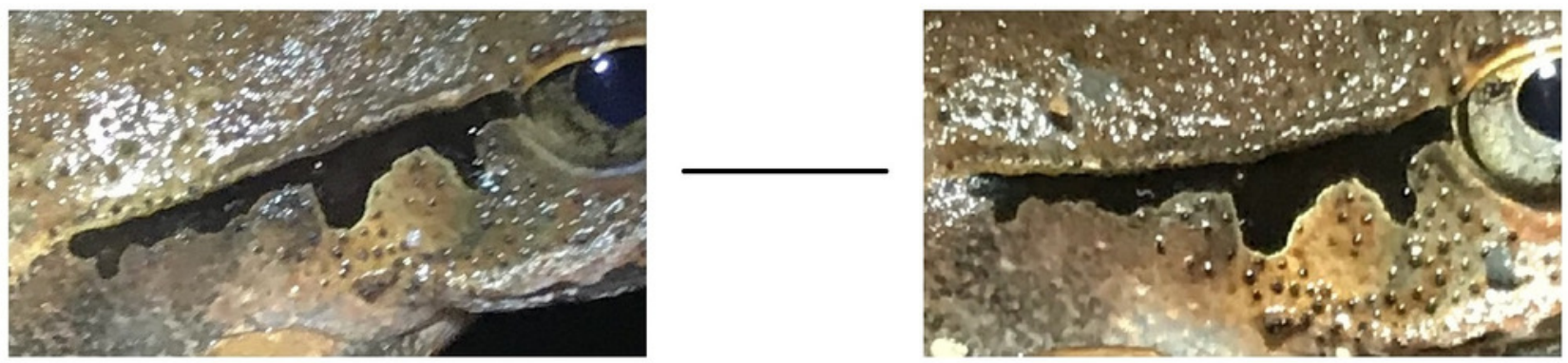

B)
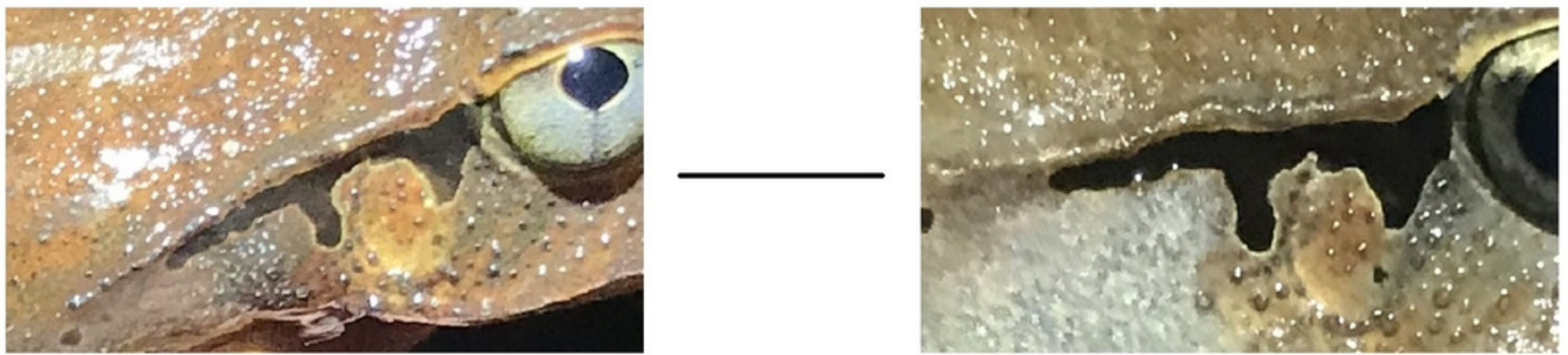

C)
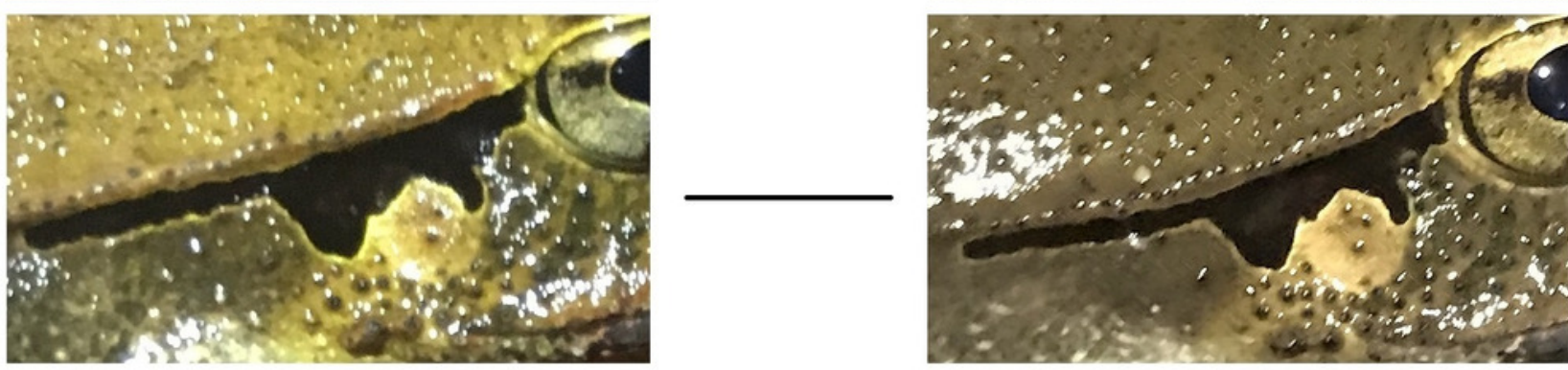

D)
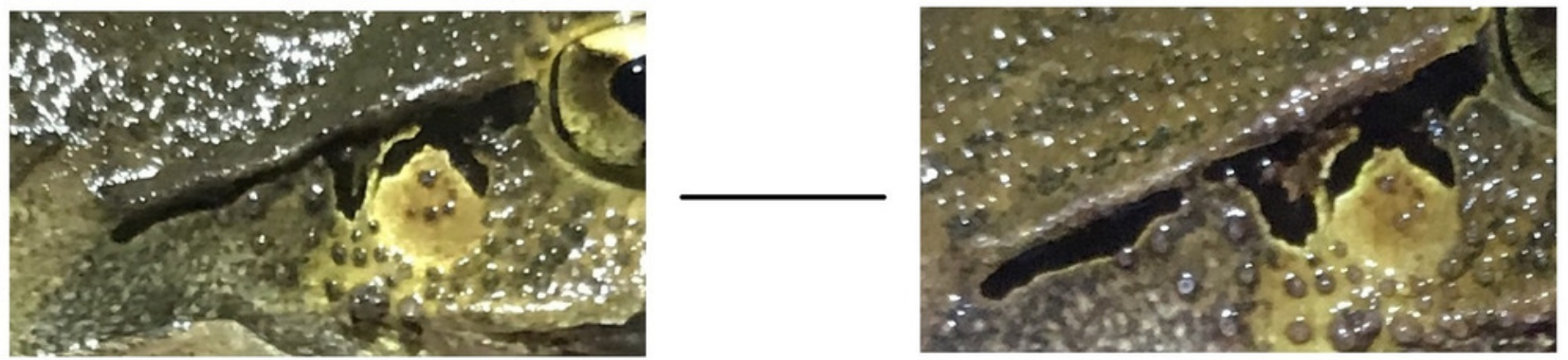
Figure 4

Frequency distribution of the number of query images (30 questions in total) correctly matched per survey event.

Note the strong left-skewed distribution, showing participants matched most queries images correctly.

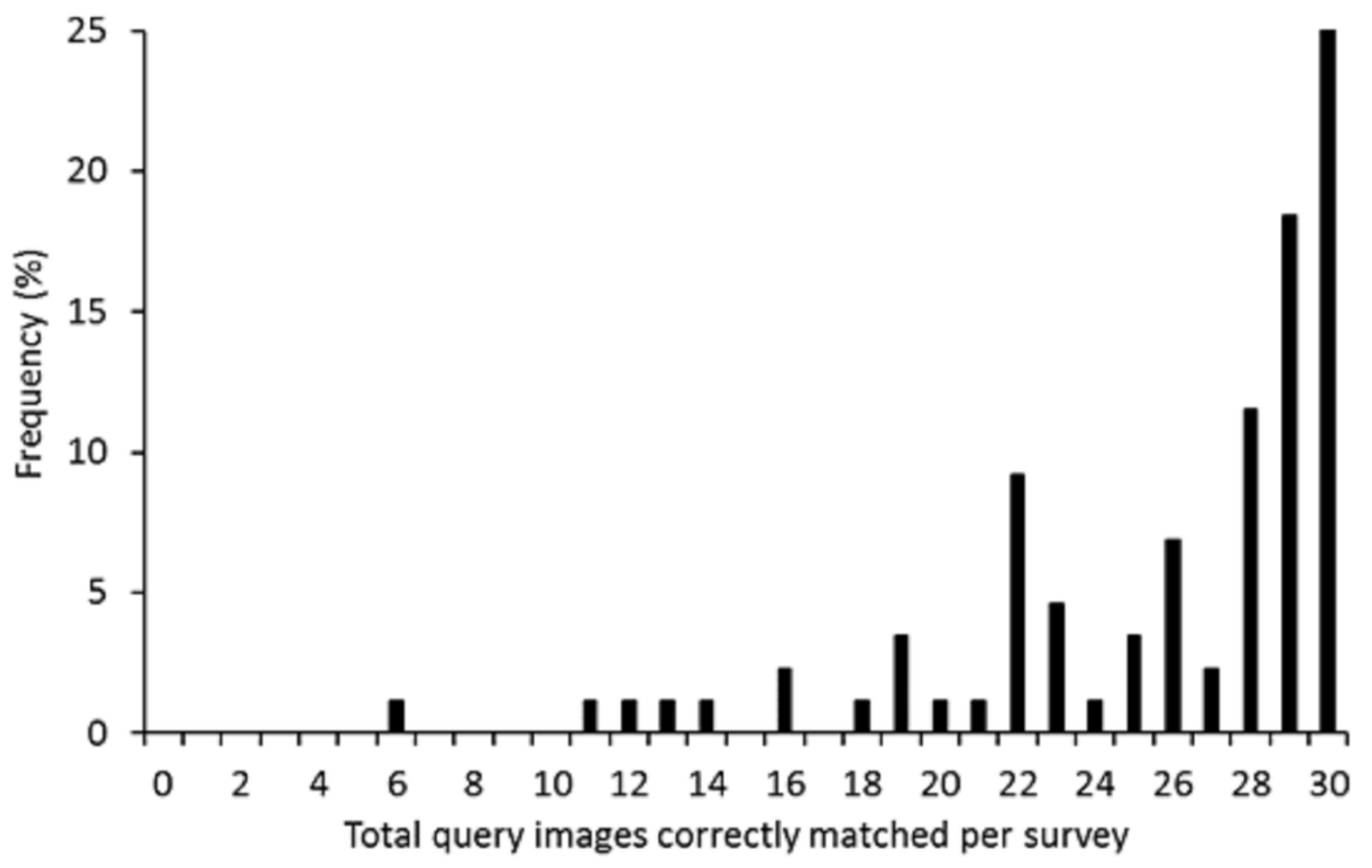




\section{Figure 5}

Samples of question and answer images from adult Lechriodus fletcheri used in the online photo-matching survey.

Participants were asked to match a query image of an unknown individual (above the line) with another image taken of the individual at a different point from an image pool of six possible matches (below the line). The correct match for the query individual is the first image below the line, although the order was randomized within questions for the surveys. Samples are from individuals correctly matched often (frog $A=99 \%$ and frog $B=97 \%$ ), or less often (frog $C=68 \%$, and frog $D=66 \%$ ) by participants. 
A)
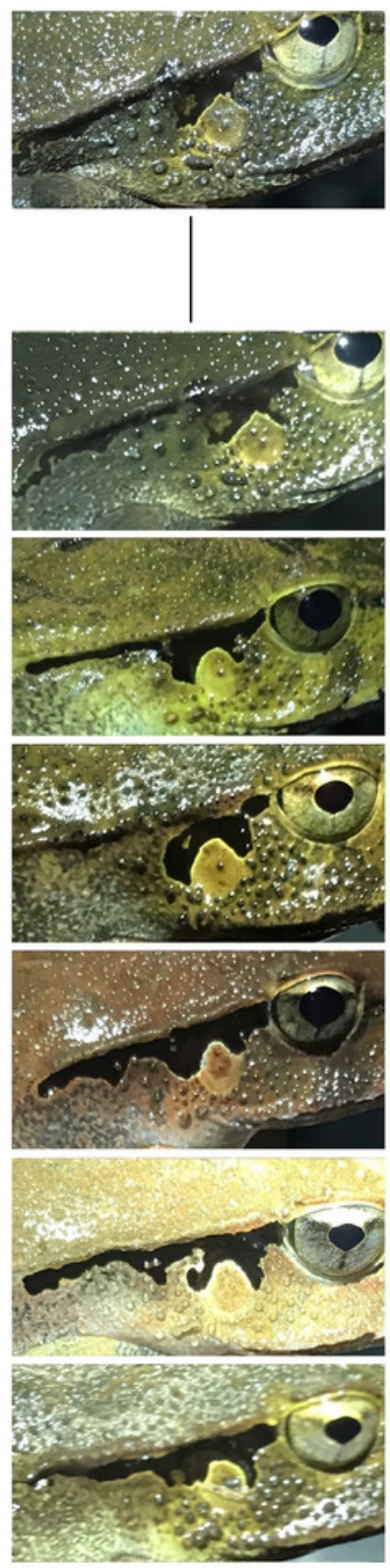

B)
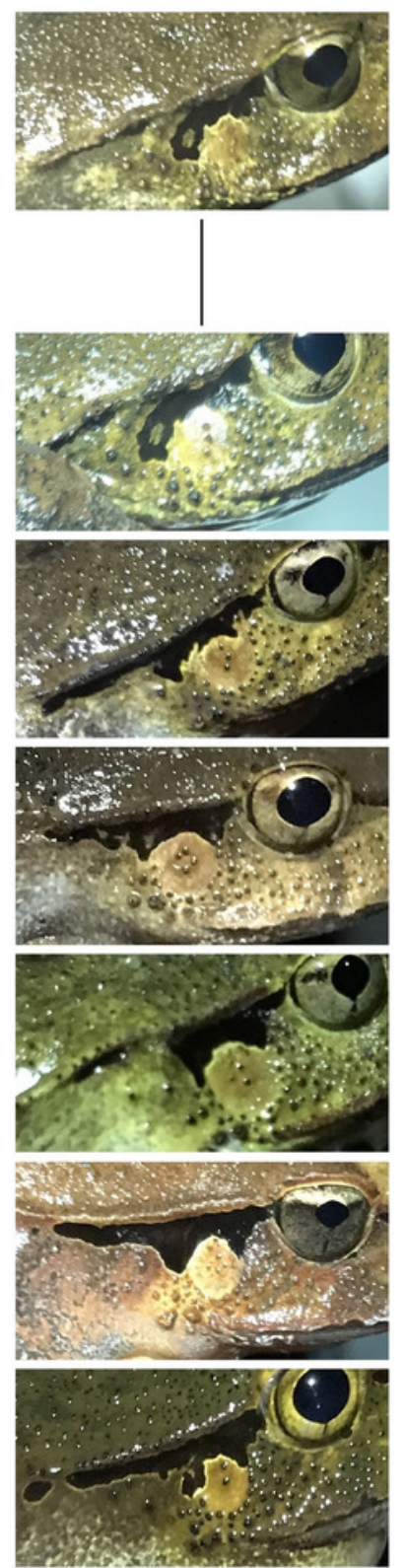

C)
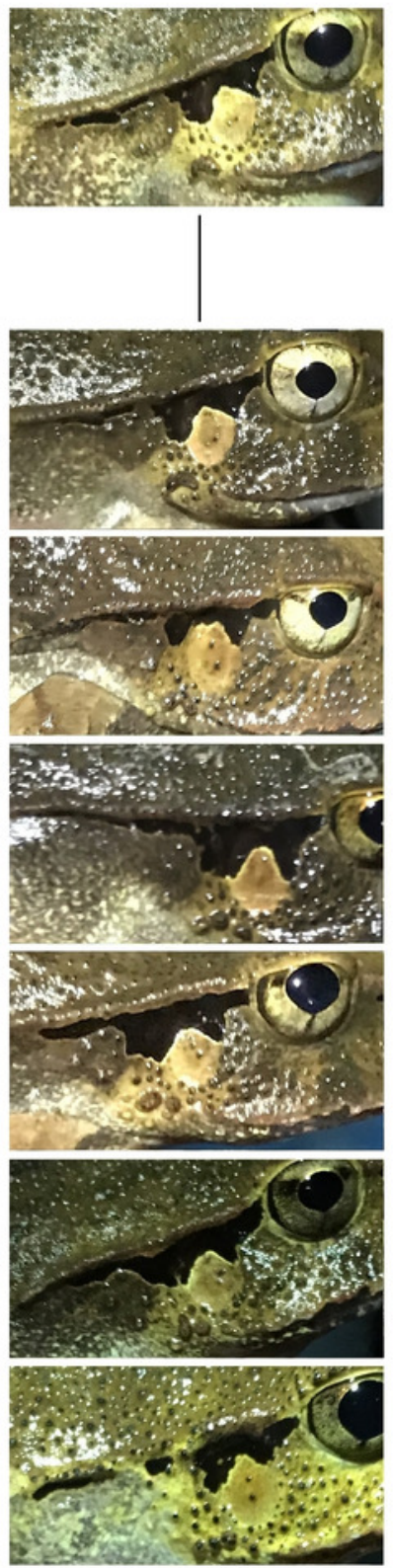

D)
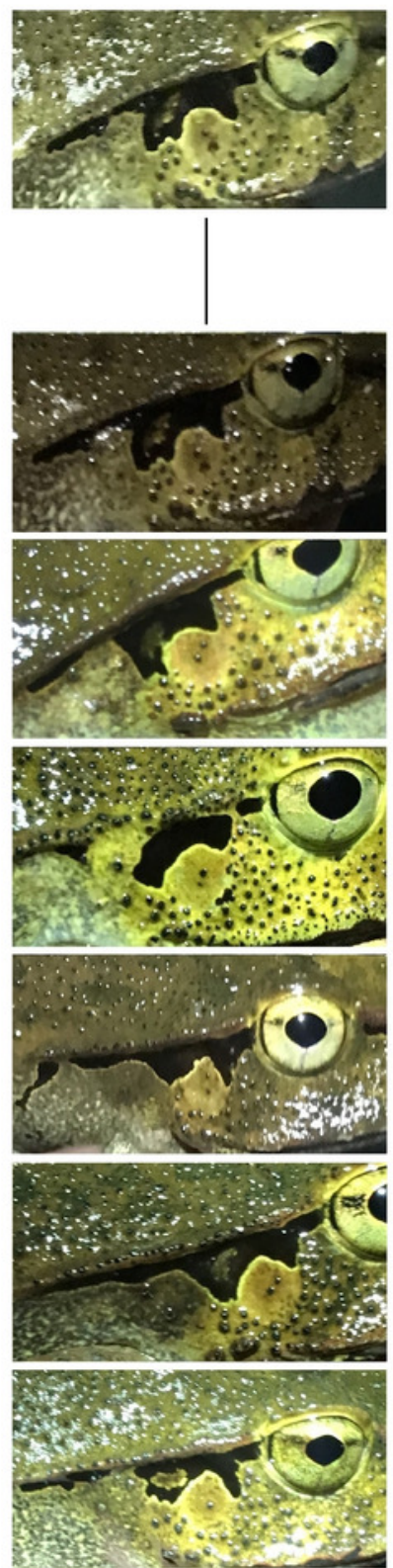